Author affiliations appear at the end of this article.

tDeceased

Published online ahead of print at www.jco.org on February 16, 2016.

Written on behalf of the Italian Lymphoma Foundation.

Supported by the Italian Lymphoma Foundation.

Authors' disclosures of potential conflicts of interest are found in the article online at www.jco.org. Author contributions are

found at the end of this article.

Clinical trial information: EudraCT 2008002684-14 and NCT 00784537.

Corresponding author: Pier Luigi Zinzani, MD, Institute of Hematology L. e A. Seràgnoli, Sant'Orsola-Malpighi University Hospital, University of Bologna, Via Massarenti, 9-40138 Bologna, Italy: e-mail: pierluigi.zinzani@unibo.it.

C 2016 by American Society of Clinical Oncology

$0732-183 X / 16 / 3412 w-1376 w / \$ 20.00$

DOI: $10.1200 / J C O .2015 .63 .0699$

\title{
Interim Positron Emission Tomography Response-Adapted Therapy in Advanced-Stage Hodgkin Lymphoma: Final Results of the Phase II Part of the HD0801 Study
}

Pier Luigi Zinzani, Alessandro Broccoli, Daniela Maria Gioia, Antonio Castagnoli, Giovannino Ciccone, Andrea Evangelista, Armando Santoro, Umberto Ricardi, Maurizio Bonfichi, Ercole Brusamolino, $\dagger$ Giuseppe Rossi, Antonella Anastasia, Francesco Zaja, Umberto Vitolo, Vincenzo Pavone, Alessandro Pulsoni, Luigi Rigacci, Gianluca Gaidano, Caterina Stelitano, Flavia Salvi, Chiara Rusconi, Monica Tani, Roberto Freilone, Patrizia Pregno, Eugenio Borsatti, Gian Mauro Sacchetti, Lisa Argnani, and Alessandro Levis

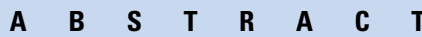

\section{Purpose}

The clinical impact of positron emission tomography (PET) evaluation performed early during firstline therapy in patients with advanced-stage Hodgkin lymphoma, in terms of providing a rationale to shift patients who respond poorly onto a more intensive regimen (PET response-adapted therapy), remains to be confirmed.

\section{Patients and Methods}

The phase II part of the multicenter HD0801 study involved 519 patients with advanced-stage de novo Hodgkin lymphoma who received an initial treatment with doxorubicin, bleomycin, vinblastine, and dacarbazine (ABVD) and who underwent an early ifosfamide-containing salvage treatment followed by stem-cell transplantation if they showed a positive PET evaluation after two cycles of chemotherapy (PET2). The primary end point was 2-year progression-free survival calculated for both PET2-negative patients (who completed a full six cycles of ABVD treatment) and PET2-positive patients. Overall survival was a secondary end point.

\section{Results}

In all, 103 of the 512 evaluable patients were PET2 positive. Among them, 81 received the scheduled salvage regimen with transplantation, 15 remained on ABVD (physician's decision, mostly because of minimally positive PET2), five received an alternative treatment, and two were excluded because of diagnostic error. On intention-to-treat analysis, the 2-year progression-free survival was $76 \%$ for PET2-positive patients (regardless of the salvage treatment they received) and $81 \%$ for PET2negative patients.

\section{Conclusion}

Patients with advanced-stage Hodgkin lymphoma for whom treatment was at high risk of failing appear to benefit from early treatment intensification with autologous transplantation, as indicated by the possibility of successful salvage treatment in more than $70 \%$ of PET2-positive patients through obtaining the same 2-year progression-free survival as the PET2-negative subgroup.

\section{J Clin Oncol 34:1376-1385. (C) 2016 by American Society of Clinical Oncology}

\section{INTRODUCTION}

Recently, several studies have clearly demonstrated the predictive value of a positron emission tomography (PET) scan performed after two cycles of chemotherapy (ie, interim or PET2) in patients with advanced-stage Hodgkin lymphoma (HL). ${ }^{1-6}$ In parallel, large cohort studies have pointed out several well-established pretreatment prognostic factors, although it is still the disease stage that largely determines the initial treatment strategy. ${ }^{7-9}$
Approximately $65 \%$ to $70 \%$ of patients with advanced-stage HL can be cured with six to eight cycles of doxorubicin, bleomycin, vinblastine, and dacarbazine (ABVD), with or without consolidation radiotherapy. ${ }^{10,11}$ Escalated bleomycin, etoposide, doxorubicin, cyclophosphamide, vincristine, procarbazine, and prednisone (BEACOPP) cures around $85 \%$ of patients if given as first-line therapy, but concerns regarding its acute toxicity and the possible onset of second myeloid neoplasia are limiting factors for its widespread use. ${ }^{12,13}$ 
Despite an improvement in the therapeutic management of patients with HL, early identification of those patients in whom the chances of cure by conventional treatment are low and for whom an escalated BEACOPP or an intensified high-dose regimen with a subsequent peripheral stem-cell autograft can be justified is still a crucial problem. As it happens with early-stage disease, pretreatment prognostic tools for advanced-stage disease, notably the International Prognostic Score (IPS), do not accurately predict which patients will likely receive a benefit from more intensive therapy. ${ }^{8} \mathrm{~A}$ negative interim PET scan is a strong predictor of favorable outcomes with standard therapy: long-term progression-free survival (PFS) rates of approximately $95 \%$ have been consistently shown in patients with advanced-stage HL treated with ABVD who display an early PET negativity. ${ }^{1-6}$ Consequently, patients with a poor prognosis can be identified according to their early response to induction treatment, as documented by an interim PET evaluation. Therefore, a responseadapted therapy may be tailored for patients with poor response.

Several trials that take into account a PET response-adapted therapy for patients with advanced-stage HL are ongoing or have recently been completed. The common aim of these trials is to deescalate therapy in patients with a favorable early PET response or to escalate therapy in those who do not respond well or to do both. ${ }^{14-20}$ The cost-benefit ratio of two different strategies will be tested by using interim PET as a surrogate indicator for chemotherapy sensitivity. The first strategy uses the initiation of first-line therapy with the most intensive regimen (escalated BEACOPP) and then de-escalating treatment in patients with a negative interim PET result. Alternatively, a standard ABVD treatment can be started and then escalated to either BEACOPP or high-dose chemotherapy and autologous bone marrow transplantation (ABMT) only in PET2-positive patients, who can presumably benefit from an intensified treatment.

Herein we present the final results of the phase II part of an Italian multicenter trial (HD0801; Early Salvage With High-Dose Chemotherapy and Stem-Cell Transplantation in Advanced Stage Hodgkin's Lymphoma) conducted in centers adhering to the Italian Lymphoma Foundation guidelines. This phase II trial included patients with advanced-stage HL that was PET positive after two cycles of ABVD who were offered a chemotherapy salvage treatment followed by high-dose chemotherapy with autologous stem-cell support.

\section{PATIENTS AND METHODS}

\section{Study Oversight}

HD0801 was a multicenter study involving patients with newly diagnosed advanced-stage HL, all receiving first-line ABVD treatment and undergoing a PET2 evaluation. The study will produce an estimate of the efficacy of an early PET response-adapted strategy in advanced HL. In addition, this trial was designed to address two specific questions: whether an early PET-guided salvage treatment consisting of high-dose chemotherapy with a subsequent ABMT could be considered safe and effective compared with data in the literature (phase II; Fig 1, shaded area) and whether PET2-negative patients could benefit from radiotherapy consolidation for areas of bulky disease, provided they maintained PET negativity upon completion of the planned six ABVD courses (phase III; Fig 1, white area). The follow-up of the randomized comparison between patients treated with radiotherapy versus observation is still ongoing; therefore, results will be reported separately in the future.
The applied salvage treatment (phase II) was made up of four courses of ifosfamide, gemcitabine, and vinorelbine (IGEV) ${ }^{21}$ chemotherapy, which allowed stem-cell mobilization in peripheral blood with harvesting after the third course, ${ }^{22}$ and was followed by carmustine, cytarabine, etoposide, and melphalan (BEAM)-conditioned ABMT treatment if patients had obtained a PET-documented complete response (CR). In case of a positive post-IGEV PET evaluation, patients with an HLA-matched donor were scheduled to receive high-dose melphalan-conditioned ABMT followed by a reduced-intensity allogeneic transplantation; conversely, those lacking an HLA-matched donor received high-dose melphalan ABMT followed by a BEAM-conditioned treatment.

\section{Patient Enrollment and Study Conduct}

Patients age 18 to 70 years were considered eligible if they had previously untreated and histologically documented HL (with the exception of nodular lymphocyte-predominant subtype) in clinical stage IIB to IV according to Ann Arbor staging and at least one bidimensionally measurable target lesion (even if extranodal only). Patients were excluded from the study if they had a severe disease that impaired normal life, presented an active infection, or had an inadequate liver or renal function, unless this was a result of the lymphoma. Those with a history of previous malignancy (except basal cell skin carcinoma and in situ carcinoma of the cervix) were considered ineligible.

Responses were primarily evaluated by centrally reviewed PET scan after two cycles of ABVD and at the end of the scheduled treatment plan, provided that all patients had undergone a complete staging workup, including PET examination, before the start of treatment. The depth of response was graded according to the revised response criteria for malignant lymphomas. ${ }^{23}$

All local ethic committees at each center approved the study protocol and its amendments, in accordance with the Italian law and in compliance with the Declaration of Helsinki. Patients provided written informed consent before being included in the study.

\section{Central PET Review}

In this study, central PET review played a pivotal role in reducing the variability of visual scan interpretation between various readers, because treatment decisions were made on the basis of the result of PET2 scans. PET scans were interpreted according to Juweid's criteria, ${ }^{24}$ which used mediastinal uptake as a reference. Central review of all uncertain results took place within 5 days of the PET scan at a central imaging core laboratory at the University of Florence; a panel of 11 nuclear medicine physicians served as reviewers for all procedures. The reviewing process proceeded as follows: first, a local nuclear medicine physician sent the initial and interim PET scans to the core laboratory along with the available clinical information; then, an answer from five reviewers was sent to the study data center and to local physicians. In case of discrepancy between the local PET evaluation and the central review, the result of the latter was considered predominant. All PET2-positive scans were reviewed at the end of the trial and were interpreted by using the Deauville ${ }^{25}$ criteria, now regarded as the standard criteria for PET interpretation. These criteria were elaborated and published while the study was ongoing.

\section{End Points}

The primary end point of the phase II part of the study was 2-year PFS calculated from the date of the PET2 scan to the date of lymphoma progression, death as a result of any cause, or completion of the 2 years of followup. Overall survival (OS), defined as the time from PET2 scan until death as a result of any cause or the date of the last follow-up, was a secondary outcome. In addition, the estimations of OS and PFS calculated from entry into the study (registration) until the end of follow-up were provided for the entire study population. Exploratory analyses of factors predicting response and toxicity will be performed after a longer follow-up becomes available.

\section{Statistical Analysis}

The sample size of the phase II part of the trial has been estimated according to the Fleming-A'Hern design. The primary efficacy end point 


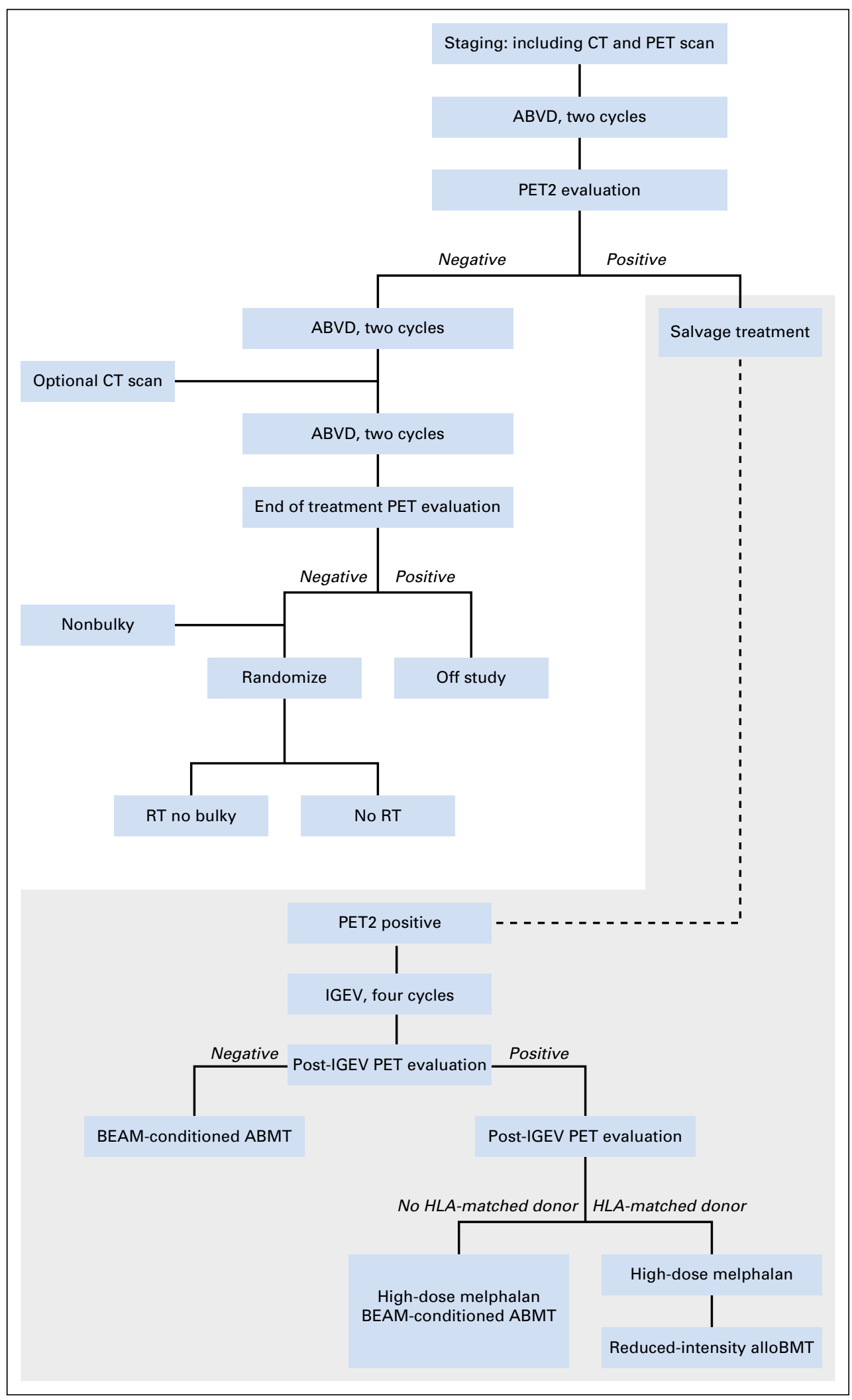

Fig 1. Study outline. The shaded area indicates the phase II part of the study. $A B M T$, autologous bone marrow transplantation; ABVD, doxorubicin, bleomycin, vinblastine, and dacarbazine; alloBMT, allogeneic bone marrow transplantation; BEAM, carmustine, cytarabine, etoposide, and melphalan; CT, computed tomography; IGEV, ifosfamide, gemcitabine, and vinorelbine; PET, positron emission tomography: PET2, PET scan performed after two cycles of chemotherapy; RT, radiotherapy.

(2-year PFS) was calculated as the cumulative proportion of patients alive and progression free at 2 years. All other time-to-event end points included in the study have been estimated by using the Kaplan-Meier method. For all point estimates, the corresponding confidence intervals were also provided.
Baseline patient characteristics were compared according to PET2 positivity by using the Mann-Whitney $U$ test and $\chi^{2}$ test (or Fisher's exact test, if appropriate) for continuous variables and categorical variables, respectively. For the safety analyses, frequency of toxicities was reported by 
type and grade according to the National Cancer Institute Common Terminology Criteria for Adverse Events (version 2.0). All analyses were performed by using STATA version 11.1 (STATA, College Station, TX).

\section{RESULTS}

In all, 520 patients who had enrolled onto the study started ABVD treatment between September 2008 and April 2013 in 50 Italian centers. Of those patients, 512 (99\%) underwent a PET2 scan, one patient withdrew consent before therapy, and seven patients interrupted the treatment before the end of the second ABVD cycle. The demographic and baseline disease characteristics for the entire population and according to PET2 evaluation are listed in Table 1.

Median age of the patients was 33 years (range, 18 to 68 years). Most of the patients (73\%) had a nodular sclerosis HL subtype, $46 \%$ presented with stage IV disease, and 35\% had bulky disease. The marrow was involved in 49 patients $(10 \%)$. An IPS $\geq 3$ was seen in 206 patients (44\%).

\section{Response to Treatment}

Among the 512 patients with an interim PET2 scan evaluated by central review, 409 (80\%) were PET negative and $103(20 \%)$ were PET positive. Once the PET2-positive scans had been reviewed and interpreted by using the Deauville 5-point scale, 70\% had a score $\geq 4$ (and 20\% had a score of 5). The remaining 30\% of PET2-positive scans received a score of 3.

As stated by the protocol, all 409 PET2-negative patients proceeded to four more ABVD cycles, whereas the remaining patients were moved to the IGEV salvage arm. However, among the 103 patients with PET2 positivity, 22 did not receive the scheduled treatment: two because of a diagnostic error and the remaining 20 because of the patients' refusal to move to high-dose treatment at that time. Therefore, the latter 20 patients continued the treatment with four more ABVD cycles (15 patients) or shifted to a different salvage regimen (five patients). More specifically, among the 15 patients who continued with ABVD therapy, nine presented with a minimally positive PET2 scan (ie, just above background, which was compatible with a Deauville score of 3 upon central revision of the cases, as stated above in Patients and Methods), and one patient had a false-positive result, as demonstrated by a negative lymph node biopsy. One patient refused the high-dose treatment and the transplantation procedure.

Regarding the other 81 PET2-positive patients, all received four courses of IGEV and were able to provide peripheral blood stem cells, which allowed them to proceed to subsequent high-dose chemotherapy with autologous stem-cell support. At the end of IGEV therapy, 43 patients were PET-negative and underwent BEAM-conditioned ABMT therapy. Among the 38 patients with a positive post-IGEV scan, 24 received tandem ABMT therapy, and 11 of them had a PET-negative result at the end of treatment. Conversely, 14 patients underwent allogeneic transplantation, and

\begin{tabular}{|c|c|c|c|c|}
\hline Characteristic & $\begin{array}{c}\text { PET2 Negative } \\
(n=409), \text { No. }(\%)\end{array}$ & $\begin{array}{c}\text { PET2 Positive } \\
(n=103) \text {, No. }(\%)\end{array}$ & $\begin{array}{c}\text { Total } \\
(n=512), \text { No. }(\%)\end{array}$ & $P$ \\
\hline Median age & 33 & 32 & 33 & .198 \\
\hline Male sex & $222(54)$ & $53(51)$ & $275(54)$ & .608 \\
\hline IPS* & & & & .996 \\
\hline $0-2$ & $209(56)$ & $52(56)$ & $261(56)$ & \\
\hline$\geq 3$ & $165(44)$ & $41(44)$ & $206(44)$ & \\
\hline Histology & & & & .520 \\
\hline Nodular sclerosis & $292(71)$ & $80(78)$ & $372(73)$ & \\
\hline Mixed cellularity & $60(15)$ & $11(11)$ & $71(14)$ & \\
\hline Lymphocyte depletion & $10(2)$ & $1(1)$ & $11(2)$ & \\
\hline Lymphocyte rich & $18(4)$ & $2(2)$ & $20(4)$ & \\
\hline Unspecified & $29(7)$ & $9(9)$ & $38(7)$ & \\
\hline No systemic symptoms & $144(35)$ & $42(41)$ & $186(36)$ & .294 \\
\hline B symptoms & $265(65)$ & $61(59)$ & $326(64)$ & \\
\hline LDH increase & $129(32)$ & $37(36)$ & $166(32)$ & .396 \\
\hline Performance status & & & & .966 \\
\hline 0 & $271(66)$ & $68(66)$ & $339(66)$ & \\
\hline 1 & $112(27)$ & $30(29)$ & $142(28)$ & \\
\hline 2 & $26(6)$ & $4(4)$ & $30(6)$ & \\
\hline 3 & $0(0)$ & $1(1)$ & $1(0)$ & \\
\hline Ann Arbor stage & & & & .810 \\
\hline ॥ & $80(20)$ & $19(18)$ & $99(19)$ & \\
\hline III & $145(35)$ & 34 (33) & $179(35)$ & \\
\hline IV & $184(45)$ & $50(49)$ & $234(46)$ & \\
\hline Bulky disease & $143(35)$ & $38(37)$ & $181(35)$ & .714 \\
\hline Extranodal involvement & $182(44)$ & $54(52)$ & $236(46)$ & .149 \\
\hline Marrow involvement† & 37 (9) & $12(12)$ & $49(10)$ & .454 \\
\hline
\end{tabular}


four were PET negative after treatment. Twenty-three patients (28\%) were still PET-positive at the end of the entire therapeutic course (as can be extrapolated from Fig 2).

Among the 15 patients who completed the induction phase with ABVD, 11 (73\%) obtained a CR; four patients achieved the CR after consolidation radiotherapy. One patient achieved a partial response (PR) and then received high-dose chemotherapy with stem-cell rescue and obtained a final CR; the remaining three patients relapsed or progressed. No follow-up data are available for the five patients who were shifted to a different treatment plan because they withdrew consent for the study.

Patients were compliant with follow-up procedures, and all scheduled computed tomography (CT) and PET scans were performed at the correct time points, with a tolerance of 7 days. No PET protocol violations occurred.

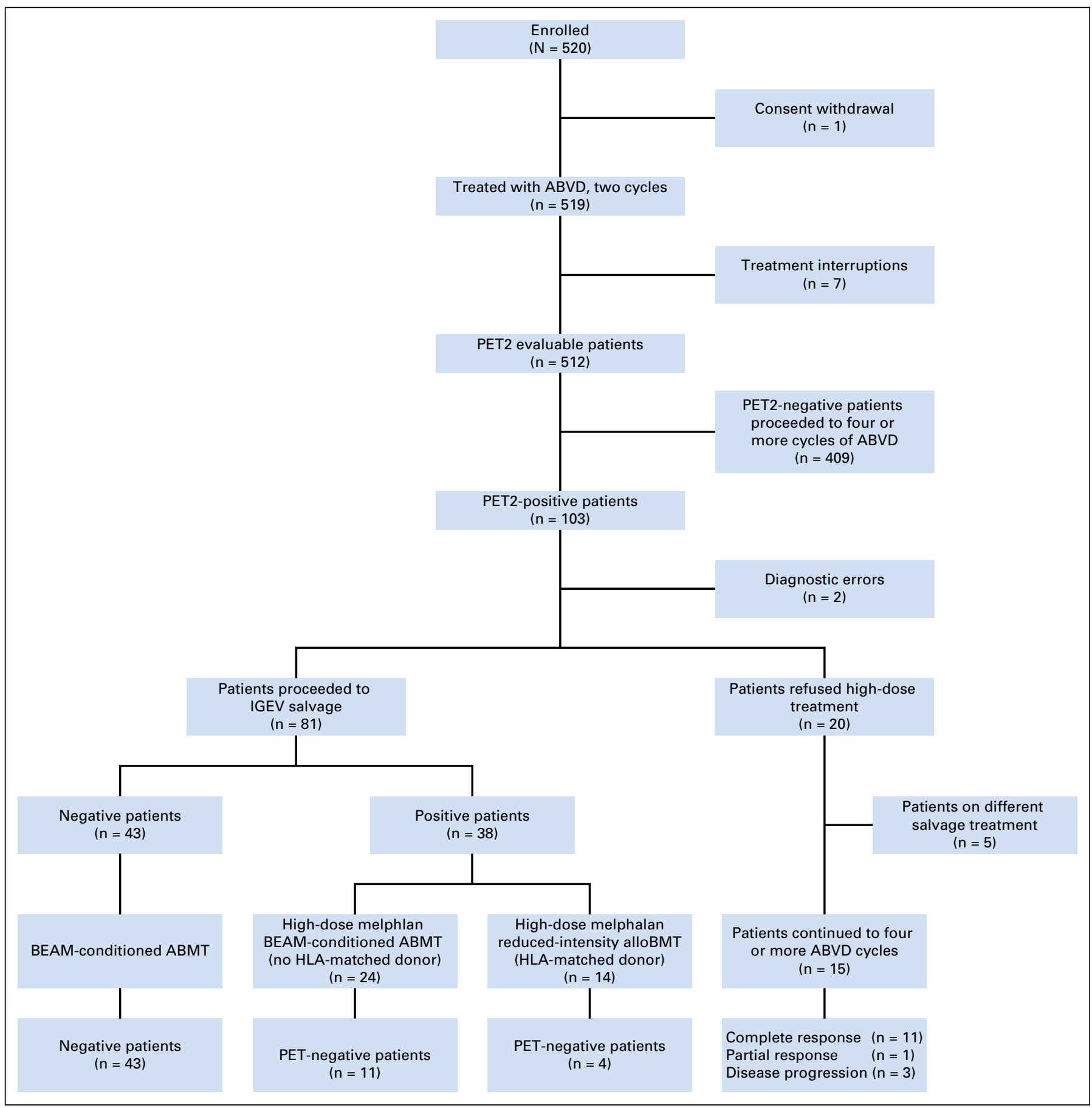

Fig 2. Patient flow diagram. ABMT, autologous bone marrow transplantation; $A B V D$, doxorubicin, bleomycin, vinblastine, and dacarbazine; alloBMT, allogeneic bone marrow transplantation; BEAM, carmustine, cytarabine, etoposide, and melphalan; IGEV, ifosfamide, gemcitabine, and vinorelbine; PET2, positron emission tomography scan performed after two cycles of chemotherapy. 


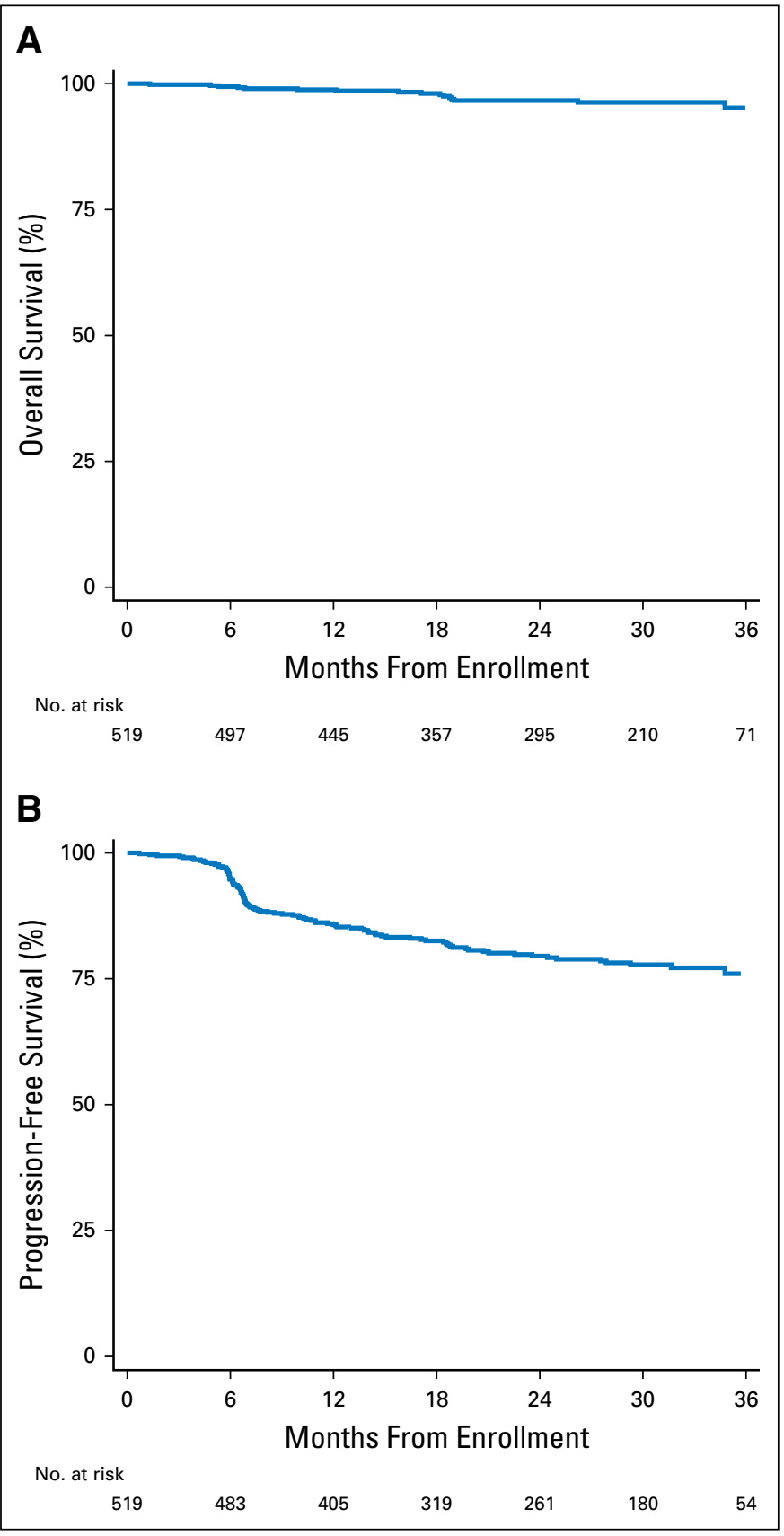

Fig 3. (A) Overall survival and (B) progression-free survival for the entire study population, as determined from the time of enrollment.

\section{Survival Analysis}

Overall, 97 patients experienced disease progression (21 in the PET2-positive group, 73 in the PET2-negative group, three in the group of patients without PET2), and 18 patients died (eight in the PET2-positive group: lymphoma $[\mathrm{n}=4]$, heart failure $[\mathrm{n}=1]$, viral encephalitis $[n=1]$, graft-versus-host disease $[n=2]$; nine in the PET2-negative group: lymphoma $[\mathrm{n}=3]$, pneumonia $[\mathrm{n}=1]$, septic shock $[\mathrm{n}=1]$, lung infection $[\mathrm{n}=2]$, secondary neoplasm $[\mathrm{n}=$ $1]$, graft-versus-host disease [n=1]; and one in the group of patients without a PET2 scan who died as a result of lymphoma).

After a median follow-up of 27 months from enrollment, the Kaplan-Meier estimates for the entire population (calculated from

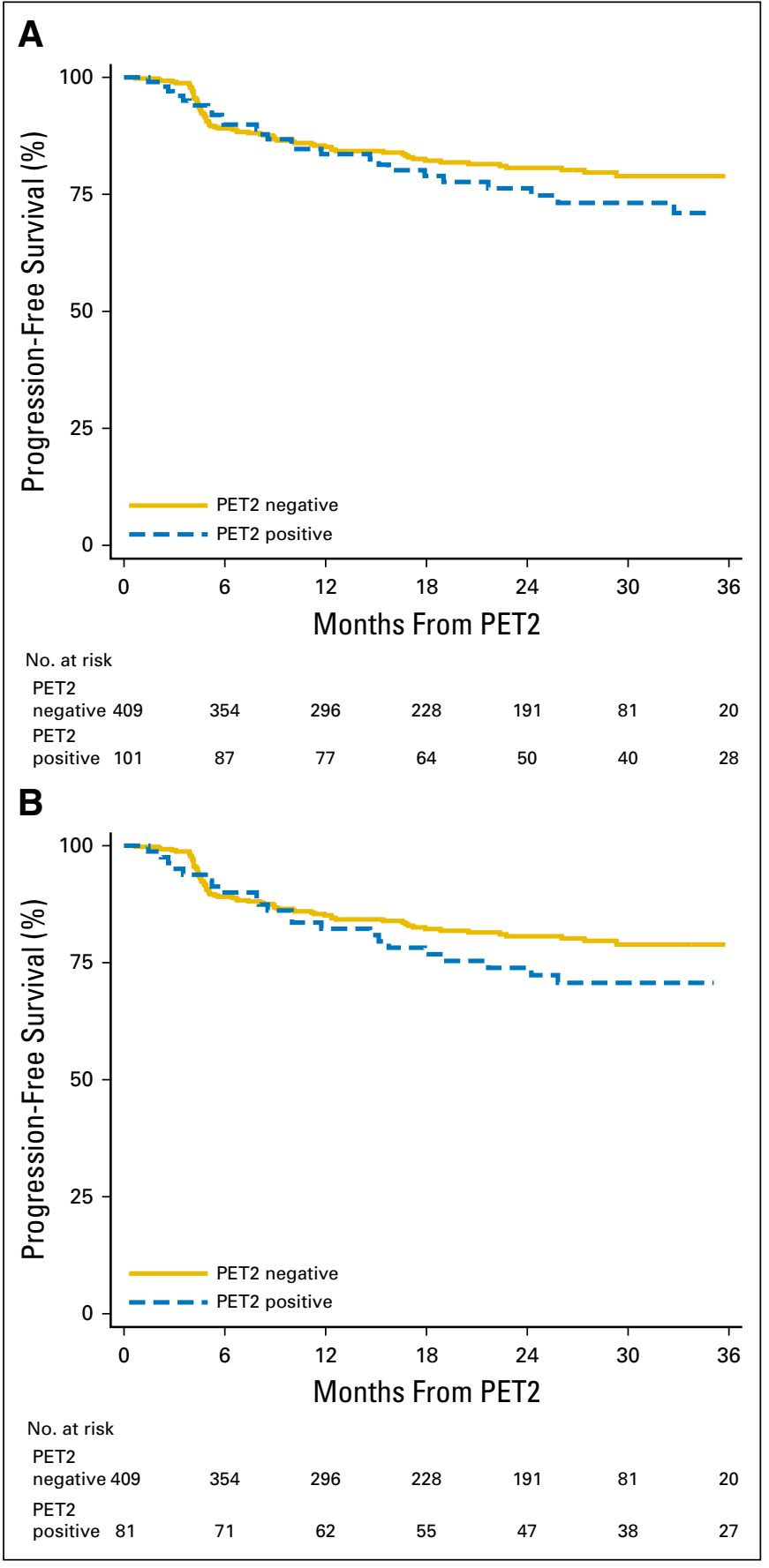

Fig 4. (A) Progression-free survival on an intention-to-treat basis for PET 2-positive (dashed line; $n=101$ ) and PET2-negative (solid line; $n=409$ ) patients who received either IGEV chemotherapy and transplantation or an alternative salvage treatment (including four more ABVD cycles). (B) Progression-free survival for PET2-negative patients ( $n=409$, solid line), all of whom were scheduled to receive six cycles of ABVD, compared with PET2-positive patients who actually received IGEV chemotherapy and transplantation ( $n=81$, dashed line). ABVD, doxorubicin, bleomycin, vinblastine, and dacarbazine; IGEV, ifosfamide, gemcitabine, and vinorelbine; PET2, positron emission tomography scan performed after two cycles of chemotherapy.

entry onto the trial) are $97 \%$ (95\% CI, $94 \%$ to $98 \%$ ) for 2-year OS (Fig $3 \mathrm{~A}$ ) and $80 \%$ (95\% CI, 76\% to $83 \%$ ) for 2-year PFS (Fig 3B). On an intention-to-treat analysis, after a median follow-up of 25 months from PET2 scanning, the 2-year PFS for the PET2-negative patients planned to receive six courses of ABVD was $81 \%$ ( $95 \%$ CI, $76 \%$ to 


\begin{tabular}{|c|c|c|c|c|c|c|c|c|c|c|}
\hline \multirow[b]{3}{*}{ Toxicity } & \multicolumn{10}{|c|}{ Grade } \\
\hline & \multicolumn{2}{|c|}{0} & \multicolumn{2}{|c|}{1} & \multicolumn{2}{|c|}{2} & \multicolumn{2}{|c|}{3} & \multicolumn{2}{|c|}{4} \\
\hline & No. & $\%$ & No. & $\%$ & No. & $\%$ & No. & $\%$ & No. & $\%$ \\
\hline Hematologic & 16 & 20 & 1 & 1 & 6 & 7 & 7 & 9 & 51 & 63 \\
\hline Granulocytes & 19 & 23 & - & - & 4 & 5 & 9 & 11 & 49 & 60 \\
\hline Hemoglobin & 19 & 23 & 15 & 19 & 33 & 41 & 6 & 7 & 8 & 10 \\
\hline Platelets & 18 & 22 & 4 & 5 & 7 & 9 & 12 & 15 & 40 & 49 \\
\hline WBC & 19 & 23 & - & - & 6 & 7 & 8 & 10 & 48 & 59 \\
\hline Nonhematologic & 20 & 25 & 5 & 6 & 15 & 19 & 37 & 46 & 4 & 5 \\
\hline Cardiac & 81 & 100 & - & - & - & - & - & - & - & 一 \\
\hline Supraventricular arrhythmia & 81 & 100 & - & - & - & - & - & - & - & - \\
\hline Ventricular arrhythmia & 81 & 100 & - & - & - & - & - & - & - & - \\
\hline Ischemia/infarct & 81 & 100 & - & - & - & - & - & - & - & 一 \\
\hline Hypertension & 81 & 100 & - & - & - & - & - & - & - & - \\
\hline Hypotension & 81 & 100 & - & - & - & - & - & - & - & 一 \\
\hline Pericarditis & 81 & 100 & - & - & - & - & - & - & - & - \\
\hline Pulmonary hypertension & 81 & 100 & - & - & - & - & - & - & - & - \\
\hline Valvular defects & 81 & 100 & - & - & - & - & - & - & - & - \\
\hline Febrile neutropenia & 55 & 68 & - & - & - & - & 25 & 31 & 1 & 1 \\
\hline Gastrointestinal & 36 & 44 & 10 & 12 & 20 & 25 & 14 & 17 & 1 & 1 \\
\hline Constipation & 76 & 94 & 4 & 5 & 1 & 1 & - & - & - & - \\
\hline Diarrhea & 65 & 80 & 4 & 5 & 9 & 11 & 3 & 4 & - & - \\
\hline Mucosal & 36 & 44 & 10 & 12 & 21 & 26 & 13 & 16 & 1 & 1 \\
\hline Hemorrhagic toxicity & 79 & 98 & 1 & 1 & - & - & 1 & 1 & - & 一 \\
\hline Hepatic and/or pancreatic & 74 & 91 & 1 & 1 & 4 & 5 & 1 & 1 & 1 & 1 \\
\hline Hepatic dysfunction & 74 & 91 & 1 & 1 & 4 & 5 & 1 & 1 & 1 & 1 \\
\hline Pancreatitis & 81 & 100 & - & - & - & - & - & - & - & - \\
\hline Infective & 59 & 73 & 2 & 2 & 7 & 9 & 11 & 14 & 2 & 2 \\
\hline Bacterial & 62 & 77 & 1 & 1 & 6 & 7 & 10 & 12 & 2 & 2 \\
\hline Fungal & 78 & 96 & 1 & 1 & 1 & 1 & 1 & 1 & - & 一 \\
\hline Viral & 75 & 93 & 2 & 2 & 3 & 4 & 1 & 1 & - & - \\
\hline Metabolic & 80 & 99 & 1 & 1 & - & - & - & - & - & - \\
\hline Hyperbilirubinemia & 81 & 100 & - & - & - & - & - & - & - & - \\
\hline Hyperglycemia & 80 & 99 & 1 & 1 & - & - & - & - & - & - \\
\hline Hyperuricemia & 81 & 100 & - & - & - & - & - & - & - & 一 \\
\hline Hypoglycemia & 81 & 100 & - & - & - & - & - & - & - & - \\
\hline Neurologic & 81 & 100 & - & - & - & - & - & - & - & - \\
\hline Cranial nerve neuropathy & 81 & 100 & - & - & - & - & - & - & - & - \\
\hline Cerebrovascular ischemia & 81 & 100 & - & - & - & - & - & - & - & - \\
\hline Motor neuropathy & 81 & 100 & - & - & - & - & - & - & - & 一 \\
\hline Pulmonary & 78 & 96 & 1 & 1 & - & - & 2 & 2 & - & 一 \\
\hline Dyspnea & 78 & 96 & 1 & 1 & - & - & 2 & 2 & - & - \\
\hline Pulmonary fibrosis & 80 & 99 & & & - & - & 1 & 1 & - & - \\
\hline Renal failure & 78 & 96 & - & - & 3 & 4 & - & - & - & - \\
\hline Vascular & 79 & 98 & - & - & 1 & 1 & 1 & 1 & - & - \\
\hline Phlebitis & 80 & 99 & - & - & 1 & 1 & - & - & - & - \\
\hline Thrombosis/embolism & 80 & 99 & - & - & - & - & 1 & 1 & - & - \\
\hline Other toxicities & 64 & 79 & 5 & 6 & 9 & 11 & 1 & 1 & 2 & 2 \\
\hline
\end{tabular}

84\%), whereas the 2-year PFS for the PET2-positive patients ( $\mathrm{n}=101$, independently from the IGEV, ABVD, or other salvage treatment they received) was $76 \%$ (95\% CI, $66 \%$ to $84 \%$; Fig $4 \mathrm{~A})$. When calculated for only the 81 PET2-positive patients who received IGEV salvage treatment, the 2-year PFS was $74 \%$ (95\% CI, $62 \%$ to $82 \%$; Fig $4 \mathrm{~B}$ ).

In an intention-to-treat analysis (independently from the salvage treatment patients received), we compared PET-negative patients with PET-positive patients excluding those with Deauville score 3: the 2-year PFS of the PET-positive group was 75\% (95\% CI, 57\% to 86\%; Fig 5).

\section{Toxicity}

Among the 81 patients who received the scheduled salvage procedure, grade 3 and 4 adverse events were primarily hematologic and directly correlated with the treatment itself. Grade 3 and 4 neutropenia was documented in $11 \%$ and $60 \%$ of patients, respectively, and grade 3 and 4 thrombocytopenia was seen in 15\% and $49 \%$ of patients, respectively. The incidence of severe anemia was lower, with grade 3 in $7 \%$ of the patients and grade 4 in 10\%; $41 \%$ of the patients had grade 2 anemia. No treatment toxicityrelated hospitalization or treatment-related deaths occurred.

The most significant grade 3 and 4 extrahematologic adverse events (documented in at least $10 \%$ of the patients) were febrile neutropenia (grade 3; 31\%), mucositis (grade 3; 16\%), and bacterial infections (grade $3 ; 12 \%$ ); all patients recovered rapidly. All the other grade 3 and 4 adverse events were rare: one grade 4 hepatic dysfunction and eight grade 3 events (diarrhea $[\mathrm{n}=3]$, dyspnea [ $\mathrm{n}=2]$, hemorrhagic toxicity $[\mathrm{n}=1]$, pulmonary fibrosis $[\mathrm{n}=1]$, and 
thrombosis $[\mathrm{n}=1]$ ). The remaining toxic effects were mild (grade 1 and 2) and transient. A complete list of toxic events is provided in Table 2.

\section{DISCUSSION}

Survival rates for patients with HL, even in advanced stages, have substantially increased over the last few decades. However, a proper balance between risks and benefits of different treatment strategies has not yet been achieved, and the key question of whether to use an intensified chemotherapy for first-line treatment or to reserve it for high-risk or relapsing patients is still without an answer. The standard first-line approach is based on the ABVD regimen. ${ }^{10,11}$ A second-line treatment with high-dose chemotherapy followed by ABMT is generally reserved for the $25 \%$ of patients who relapse after initial treatment. An alternative approach consists of trying to cure as many patients as possible with a more aggressive regimen (ie, escalated BEACOPP), to be used from the beginning. Systematic review and network meta-analysis have shown better PFS and OS rates, although this more intense approach exposes patients to considerable acute and late chemotherapy-related toxicity. ${ }^{12,13,26} \mathrm{~A}$ randomized comparison of ABVD and BEACOPP in patients with advanced-stage $\mathrm{HL}$ has recently been reported, ${ }^{10}$ and its results have led some authors to conclude that initial therapy may not necessarily be highly aggressive in all patients because those who relapse may receive subsequent intensive salvage therapy. Others have pointed out that OS was a secondary end point in this study and that the study was small compared with other similar trials. ${ }^{27}$ At this time, physicians are not able to predetermine which patients can be cured by ABVD and which patients will benefit from escalated BEACOPP.

PET is now considered an essential component in HL management (because it is widely used for disease staging, restaging, and response evaluation), and the results of an interim PET assessment (generally after the first two cycles of chemotherapy) may be regarded as a strong predictor of the final outcome..$^{2-4}$ This has been proven in several studies in which a PET scan performed after one to three cycles of chemotherapy (ABVD was used in all studies) reliably predicted the treatment outcome in more than $85 \%$ to $90 \%$ of patients with HL. ${ }^{1-6}$ Nevertheless, the clinical impact of the interim response assessment during therapy (in other words, if a positive interim PET could justify a shift to a more intensive treatment regimen) remains to be confirmed by the results of ongoing prospective trials. So far, only three studies have reported on the impact of PET response-adapted therapy in advanced-stage HL; however, some studies have methodologic flaws (eg, a small number of patients; use of interim ${ }^{67} \mathrm{Ga}$ scintigraphy in some patients) with the last trial being simply a retrospective analysis. $14,28,29$

What we report here are the results of the first prospective multicenter interim PET-adapted trial in a cohort of 512 patients with advanced-stage HL. The percentage of patients showing a positive PET2 (20\%) was similar to that reported in previous studies. ${ }^{4,5}$ In the cohort of PET2-negative patients, the 2-year PFS was $81 \%$ (overlapping with historical controls), whereas in PET2-positive patients, the 2-year PFS increased from $12 \%$ of the historical control to $74 \%$ (76\% on an intention-to-treat analysis) of this study. ${ }^{5,10,11,30}$ One issue in our study is the group of 15 PET2-positive patients who received four more ABVD cycles as a result of the physician's or patient's refusal to switch to the salvage treatment program (among the 15 patients, nine had a minimally positive PET2, which corresponded to Deauville score 3 upon central revision, and one had a negative biopsy of a PETpositive lymph node); 73\% achieved a CR, thus indicating the existence of a proportion of patients who can obtain a CR even though they show PET positivity at early evaluation. As reported in all published data sets, this percentage ranges between $10 \%$ and $30 \%$; in this study, it is $11 \% .^{1,2,4-6}$ However, the fact that PET results were assessed qualitatively (the protocol was designed in 2007 before the Deauville criteria were formulated ${ }^{25}$ ) and that patients with a borderline PET could have been misclassified could represent a potential limitation of this study. This is why we conducted a post hoc revision of all the PET2-positive patients using the Deauville criteria to determine whether the favorable survival outcome obtained by patients who received a transplantation depended on a higher proportion of patients with Deauville score 3 (ie, minimally positive PET 2 scan) being allocated to this arm, which in fact did not. Nearly $70 \%$ of the patients in the transplantation arm had a Deauville score $\geq 4$. An additional PFS analysis comparing PET2-negative and PET2-positive patients (excluding patients with a Deauville score of 3) reported comparable results, which did not change study outcomes.

To the best of our knowledge, these data support, for the first time, the role of early treatment intensification in a small proportion of patients considered at high risk for treatment failure and identified by PET2 positivity. This strategy is opposed to the conventional approach of submitting patients to salvage treatment only after they demonstrate resistance to first-line induction or as soon as the disease relapses. This is confirmed by the possibility of having more than $70 \%$ of the PET2-positive patients receive salvage therapy by obtaining the same 2-year PFS as the PET2-negative subgroup.

Reversible grade 3 and 4 cytopenias occurred, as previously reported. ${ }^{21,22}$ Neither treatment toxicity-related hospitalization nor treatment-related deaths have been documented so far, thus achieving a favorable toxicity profile for such an intensive therapeutic strategy.

The most relevant theoretical advantages of this approach may be represented by better long-term results because of reduced resistance to induction treatment and a consequently decreased incidence of early and late adverse effects. Conversely, the major disadvantage could be overtreatment of a minority of PET2positive patients who could benefit from continuation of the

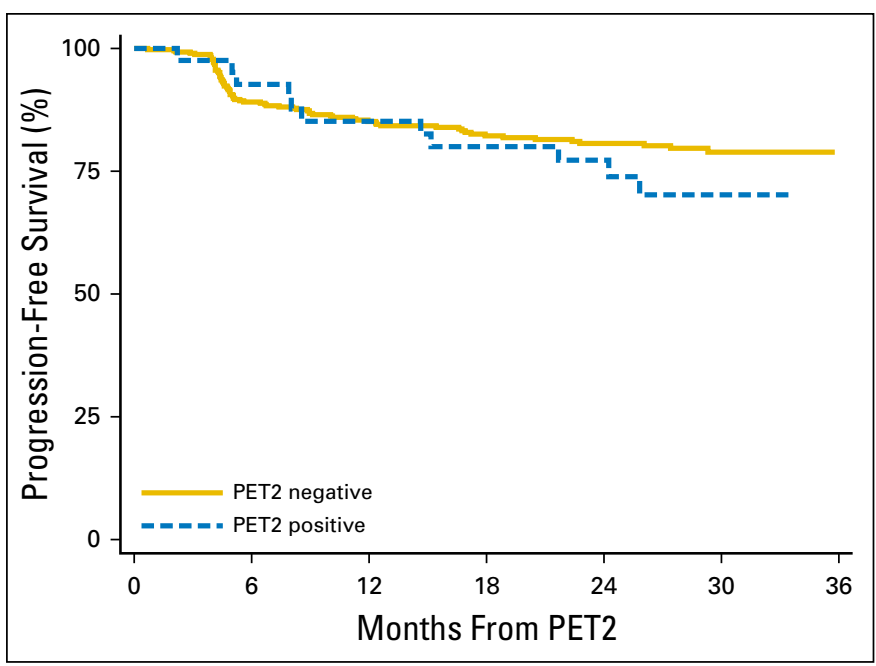

Fig 5. Progression-free survival on an intention-to-treat basis. Solid line, PET2 negative patients; dashed line, PET2-positive patients (Deauville score 4 and 5). 
original treatment plan. Longer follow-up will allow more valid and robust conclusions for long-term efficacy and toxicity.

\section{AUTHORS' DISCLOSURES OF POTENTIAL CONFLICTS} OF INTEREST

Disclosures provided by the authors are available with this article at www.jco.org.

\section{AUTHOR CONTRIBUTIONS}

Conception and design: Pier Luigi Zinzani, Alessandro Levis

Provision of study materials or patients: Pier Luigi Zinzani, Alessandro Broccoli, Daniela Maria Gioia, Antonio Castagnoli, Giovannino Ciccone,
Andrea Evangelista, Armando Santoro, Umberto Ricardi, Maurizio Bonfichi, Ercole Brusamolino, Giuseppe Rossi, Antonella Anastasia, Francesco Zaja, Umberto Vitolo, Vincenzo Pavone, Alessandro Pulsoni, Luigi Rigacci, Gianluca Gaidano, Caterina Stelitano, Flavia Salvi, Chiara Rusconi, Monica Tani, Roberto Freilone, Patrizia Pregno, Lisa Argnani, Alessandro Levis

Collection and assembly of data: Pier Luigi Zinzani, Alessandro Broccoli, Daniela Maria Gioia, Antonio Castagnoli, Giovannino Ciccone, Andrea Evangelista, Armando Santoro, Umberto Ricardi, Maurizio Bonfichi, Ercole Brusamolino, Giuseppe Rossi, Antonella Anastasia, Francesco Zaja, Umberto Vitolo, Vincenzo Pavone, Alessandro Pulsoni, Luigi Rigacci, Gianluca Gaidano, Caterina Stelitano, Flavia Salvi, Chiara Rusconi, Monica Tani, Roberto Freilone, Patrizia Pregno, Lisa Argnani, Alessandro Levis

Data analysis and interpretation: All authors

Manuscript writing: All authors

Final approval of manuscript: All authors

\section{REFERENCES}

1. Terasawa T, Lau J, Bardet S, et al: Fluorine-18fluorodeoxyglucose positron emission tomography for interim response assessment of advanced-stage Hodgkin's lymphoma and diffuse large B-cell lymphoma: A systematic review. J Clin Oncol 27: 1906-1914, 2009

2. Hutchings $M$, Loft $A$, Hansen $M$, et al: FDGPET after two cycles of chemotherapy predicts treatment failure and progression-free survival in Hodgkin lymphoma. Blood 107:52-59, 2006

3. Gallamini A, Rigacci L, Merli F, et al: The predictive value of positron emission tomography scanning performed after two courses of standard therapy on treatment outcome in advanced stage Hodgkin's disease. Haematologica 91:475-481, 2006

4. Zinzani PL, Tani M, Fanti S, et al: Early positron emission tomography (PET) restaging: A predictive final response in Hodgkin's disease patients. Ann Oncol 17:1296-1300, 2006

5. Gallamini $A$, Hutchings $M$, Rigacci $L$, et al: Early interim 2-[18F]fluoro-2-deoxy-D-glucose positron emission tomography is prognostically superior to international prognostic score in advanced-stage Hodgkin's lymphoma: A report from a joint ItalianDanish study. J Clin Oncol 25:3746-3752, 2007

6. Zinzani PL, Rigacci L, Stefoni V, et al: Early interim 18F-FDG PET in Hodgkin's Iymphoma: Evaluation on 304 patients. Eur J Nucl Med Mol Imaging 39:4-12, 2012

7. Gobbi PG, Zinzani PL, Broglia C, et al: Comparison of prognostic models in patients with advanced Hodgkin disease: Promising results from integration of the best three systems. Cancer 91: 1467-1478, 2001

8. Hasenclever D, Diehl V: A prognostic score for advanced Hodgkin's disease: International Prognostic Factors Project on Advanced Hodgkin's Disease. N Engl J Med 339:1506-1514, 1998

9. Gobbi PG, Broglia C, Di Giulio G, et al: The clinical value of tumor burden at diagnosis in Hodgkin lymphoma. Cancer 101:1824-1834, 2004

10. Viviani $S$, Zinzani $P L$, Rambaldi $A$, et al; Michelangelo Foundation; Gruppo Italiano di Terapie Innovative nei Linfomi; Intergruppo Italiano Linfomi: $A B V D$ versus BEACOPP for Hodgkin's lymphoma when high-dose salvage is planned. $\mathrm{N}$ Engl J Med 365:203-212, 2011
11. Mounier N, Brice $P$, Bologna $S$, et al; Lymphoma Study Association (LYSA): ABVD (8 cycles) versus BEACOPP (4 escalated cycles $\geq 4$ baseline): Final results in stage III-IV low-risk Hodgkin lymphoma (IPS 0-2) of the LYSA H34 randomized trial. Ann Oncol 25:1622-1628, 2014

12. Engert A, Diehl V, Franklin J, et al: Escalateddose BEACOPP in the treatment of patients with advanced-stage Hodgkin's lymphoma: 10 years of follow-up of the GHSG HD9 study. J Clin Oncol 27: 4548-4554, 2009

13. Borchmann $P$, Haverkamp $H$, Diehl $V$, et al: Eight cycles of escalated-dose BEACOPP compared with four cycles of escalated-dose BEACOPP followed by four cycles of baseline-dose BEACOPP with or without radiotherapy in patients with advanced-stage Hodgkin's lymphoma: Final analysis of the HD12 trial of the German Hodgkin Study Group. J Clin Oncol 29:4234-4242, 2011

14. Gallamini $A$, Patti $C$, Viviani $S$, et al; Gruppo Italiano Terapie Innovative nei Linfomi (GITIL): Early chemotherapy intensification with BEACOPP in advanced-stage Hodgkin lymphoma patients with a interim-PET positive after two ABVD courses. $\mathrm{Br} J$ Haematol 152:551-560, 2011

15. Dann EJ, Blumenfeld Z, Bar-Shalom R, et al: A 10-year experience with treatment of high and standard risk Hodgkin disease: Six cycles of tailored BEACOPP, with interim scintigraphy, are effective and female fertility is preserved. Am J Hematol 87 32-36, 2012

16. ClinicalTrials.gov: HD18 for advanced stages in Hodgkins lymphoma.http://www.clinicaltrials.gov/ ct2/show/NCT00515554

17. ClinicalTrials.gov: Study of a treatment driven by early PET response to a treatment not monitored by early PET in patients with AA stage 3-4 or $2 \mathrm{~B} \mathrm{HL}$ (AHL 2011).http://clinicaltrials.gov/ct2/show/NCT01358747

18. ClinicalTrials.gov: Very early FDG-PET/CTresponse adapted therapy for advanced Hodgkin lymphoma (H11).http://www.clinicaltrials.gov/ct2/show/ NCT01652261

19. ClinicalTrials.gov: S0816 Fludeoxyglucose $F$ 18-PET/CT imaging and combination chemotherapy with or without additional chemotherapy and G-CSF in treating patients with stage III or stage IV Hodgkin lymphoma.http://www.clinicaltrials.gov/ct2/show/ NCT00822120

20. ClinicalTrials.gov: Positron emission tomography (PET)-adapted chemotherapy in advanced
Hodgkin lymphoma (HL)(HD0607).http://clinicaltrials. gov/ct2/show/NCT00795613

21. Santoro A, Magagnoli $M$, Spina $M$, et al: Ifosfamide, gemcitabine, and vinorelbine: A new induction regimen for refractory and relapsed Hodgkin's lymphoma. Haematologica 92:35-41, 2007

22. Magagnoli $M$, Spina $M$, Balzarotti $M$, et al: IGEV regimen and a fixed dose of lenograstim: An effective mobilization regimen in pretreated Hodgkin's lymphoma patients. Bone Marrow Transplant 40:1019-1025, 2007

23. Cheson BD, Pfistner $B$, Juweid $M E$, et al; International Harmonization Project on Lymphoma: Revised response criteria for malignant lymphoma. $\mathrm{J}$ Clin Oncol 25:579-586, 2007

24. Juweid ME, Stroobants S, Hoekstra OS, et al; Imaging Subcommittee of International Harmonization Project in Lymphoma: Use of positron emission tomography for response assessment of lymphoma: Consensus of the Imaging Subcommittee of International Harmonization Project in Lymphoma. J Clin Oncol 25:571-578, 2007

25. Meignan $M$, Gallamini $A$, Meignan $M$, et al: Report on the first international workshop on interimPET-scan in lymphoma. Leuk Lymphoma 50: 1257-1260, 2009

26. Skoetz N, Trelle S, Rancea M, et al: Effect of initial treatment strategy on survival of patients with advancedstage Hodgkin's lymphoma: A systematic review and network meta-analysis. Lancet Oncol 14:943-952, 2013

27. Tam CS, Herschtal A, Seymour JF: ABVD versus BEACOPP for Hodgkin's lymphoma. N Engl J Med 365:1544-1545, 2011

28. Dann EJ, Bar-Shalom R, Tamir A, et al: Riskadapted BEACOPP regimen can reduce the cumulative dose of chemotherapy for standard and high-risk Hodgkin lymphoma with no impairment of outcome. Blood 109:905-909, 2007

29. Avigdor A, Bulvik S, Levi I, et al: Two cycles of escalated BEACOPP followed by four cycles of ABVD utilizing early-interim PET/CT scan is an effective regimen for advanced high-risk Hodgkin's lymphoma. Ann Oncol 21:126-132, 2010

30. Federico $M$, Luminari $S$, lannitto $E$, et al; HD2000 Gruppo Italiano per lo Studio dei Linfomi Trial: ABVD compared with BEACOPP compared with CEC for the initial treatment of patients with advanced Hodgkin's lymphoma: Results from the HD2000 Gruppo Italiano per lo Studio dei Linfomi Trial. J Clin Oncol 27:805-811, 2009 


\section{Affiliations}

Pier Luigi Zinzani, Alessandro Broccoli, and Lisa Argnani, Institute of Hematology L. e A. Seràgnoli, Sant'Orsola-Malpighi University Hospital, Bologna; Daniela Maria Gioia and Alessandro Levis, Fondazione Italiana Linfomi Onlus; Flavia Salvi, S.S. Antonio e Biagio Hospital, Alessandria; Antonio Castagnoli, Azienda Ospedaliera di Prato, Prato; Giovannino Ciccone and Andrea Evangelista, Azienda Ospedaliero-Universitaria Città della Salute e della Scienza di Torino and Centro Prevenzione Oncologica Piemonte; Umberto Ricardi, University of Turin; Umberto Vitolo and Patrizia Pregno, Azienda Ospedaliero-Universitaria Città della Salute e della Scienza di Torino; Roberto Freilone, Stabilimento Ospitaliero Ivrea, Turin; Armando Santoro, Ercole Brusamolino, and Antonella Anastasia, Istituto Clinico Humanitas; Chiara Rusconi, Azienda Ospedaliera Niguarda Cà Granda, Milan; Maurizio Bonfichi and Ercole Brusamolino, Istituto Di Ricovero e Cura a Carattere Scientifico Policlinico San Matteo di Pavia, Pavia; Giuseppe Rossi and Antonella Anastasia, Spedali Civili, Brescia; Francesco Zaja, Santa Maria della Misericordia Hospital, Udine; Vincenzo Pavone, Azienda Ospedaliera Cardinal Panico, Tricase; Alessandro Pulsoni, Sapienza University, Rome; Luigi Rigacci, Azienda Ospedaliera Careggi, Florence; Gianluca Gaidano, Amedeo Avogadro University; Gian Mauro Sacchetti, University Hospital Maggiore della Carità, Novara; Caterina Stelitano, Azienda Ospedaliera Bianchi Melacrino Morelli, Reggio Calabria; Monica Tani, Santa Maria delle Croci Hospital, Ravenna; and Eugenio Borsatti, Centro di Riferimento Oncologico, Aviano, Italy.

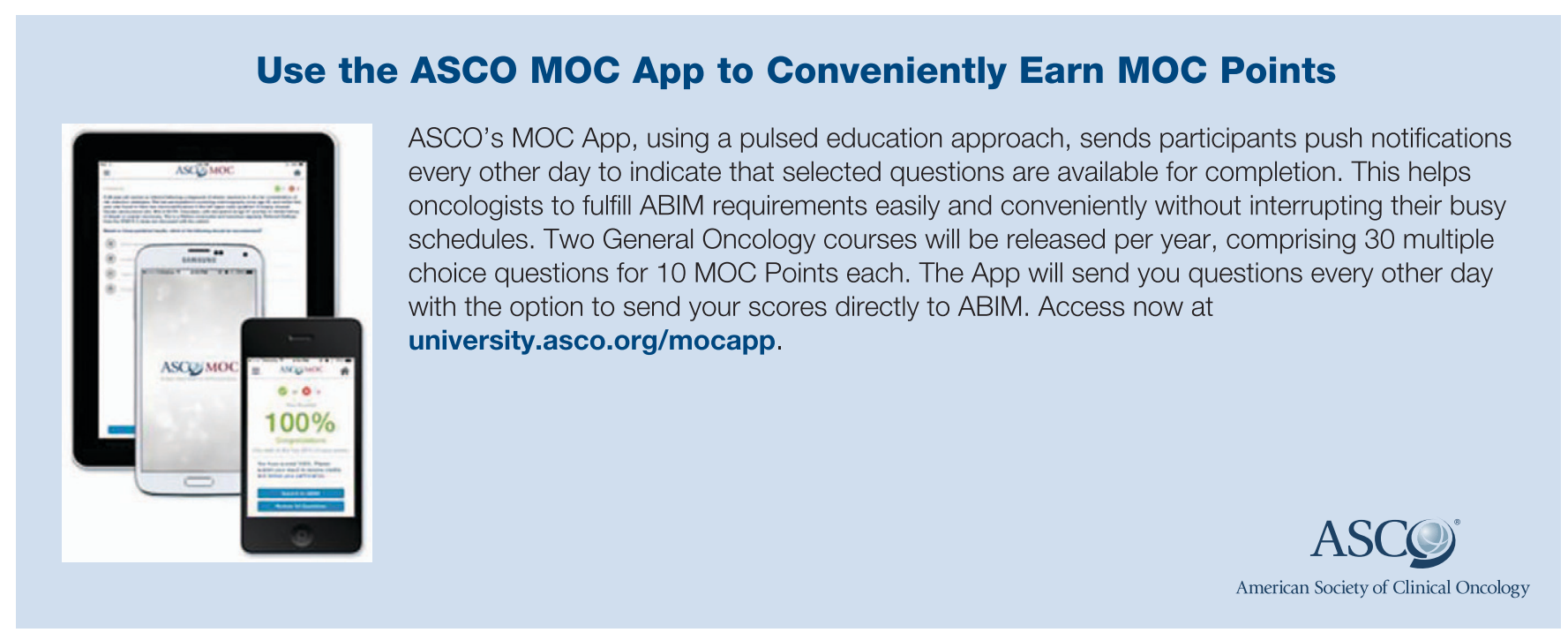


Interim Positron Emission Tomography Response-Adapted Therapy in Advanced-Stage Hodgkin Lymphoma: Final Results of the Phase II Part of the HD0801 Study

The following represents disclosure information provided by authors of this manuscript. All relationships are considered compensated. Relationships are self-held unless noted. I = Immediate Family Member, Inst = My Institution. Relationships may not relate to the subject matter of this manuscript. For more information about ASCO's conflict of interest policy, please refer to www.asco.org/rwc or jco.ascopubs.org/site/ifc.

Pier Luigi Zinzani

No relationship to disclose

Alessandro Broccoli

No relationship to disclose

Daniela Maria Gioia

No relationship to disclose

Antonio Castagnoli

No relationship to disclose

Giovannino Ciccone

No relationship to disclose

Andrea Evangelista

No relationship to disclose

Armando Santoro

No relationship to disclose

Umberto Ricardi

No relationship to disclose

Maurizio Bonfichi

Travel, Accommodations, Expenses: Takeda Pharmaceuticals, Gilead Sciences

Ercole Brusamolino

No relationship to disclose

\section{Giuseppe Rossi}

Consulting or Advisory Role: Celgene, Roche, Janssen Pharmaceuticals, Gilead Sciences, ARIAD Pharmaceuticals, Boeringher Ingelheim

\section{Antonella Anastasia}

No relationship to disclose

Francesco Zaja

No relationship to disclose

Umberto Vitolo

No relationship to disclose

Vincenzo Pavone

No relationship to disclose

\section{Alessandro Pulsoni}

No relationship to disclose

Luigi Rigacci

No relationship to disclose

\section{Gianluca Gaidano}

Honoraria: Roche, Novartis, Janssen Pharmaceuticals, GlaxoSmithKline, Amgen

Consulting or Advisory Role: Roche, Novartis, Janssen Pharmaceuticals, GlaxoSmithKline, Amgen

Travel, Accommodations, Expenses: Roche, Novartis, Janssen

Pharmaceuticals, Amgen, GlaxoSmithKline

\section{Caterina Stelitano}

No relationship to disclose

\section{Flavia Salvi}

No relationship to disclose

\section{Chiara Rusconi}

Consulting or Advisory Role: Pfizer, Janssen Pharmaceuticals

Travel, Accommodations, Expenses: TEVA Pharmaceuticals Industries, Takeda Pharmaceuticals

\section{Monica Tani}

No relationship to disclose

\section{Roberto Freilone}

No relationship to disclose

Patrizia Pregno

Consulting or Advisory Role: ARIAD Pharmaceuticals

Speakers' Bureau: Novartis, Bristol-Myers Squibb, Pfizer

\section{Eugenio Borsatti}

No relationship to disclose

Gian Mauro Sacchetti

No relationship to disclose

Lisa Argnani

No relationship to disclose

Alessandro Levis

No relationship to disclose 


\section{Acknowledgment}

We thank all the patients who participated in this study and their supportive families. We also thank the referring physicians and the supporting staff at all the participating clinical sites. We thank all the principal investigators of the other sites involved in this study: Michele Spina (Aviano), Vincenzo Liso (Bari), Giorgio Reguzzoni (Busto Arsizio), Emanuele Angelucci (Cagliari), Massimo Aglietta (Candiolo, Turin), Pier Paolo Fattori (Meldola), Giancarlo Latte (Nuoro), Anna Lia Molinari (Rimini), Elisabetta Abruzzese (Rome), Alteda Romano (Rome), Alberto Fabbri (Siena), Anna Marina Liberati (Terni), and Annalisa Luraschi (Verbania). We also wish to acknowledge all of the nuclear medicine physicians who made a great effort for the optimal conduct of this study: Alberto Pupi (Florence), Manuela Englaro (Udine), Manjola Dona (Prato), Arturo Chiti (Rozzano, Milan), Marilena Bello (Turin), Pierluigi Rossini (Brescia), and Giulia Montelatici and Enrico Pesciullesi (Arezzo).

\section{Appendix}

\section{Patients and Methods}

Patients' enrollment and study conduct. All patients had their medical history collected at the time of enrollment. Staging procedures consisted of a full physical examination; a complete blood cell count with leukocyte differential and platelet count; a computed tomography (CT) scan of neck, chest, abdomen, and pelvis (with and without contrast); an ${ }^{18} \mathrm{~F}$-fluorodeoxyglucose (FDG) positron emission tomography (PET) scan; and a bone marrow aspiration and biopsy. A mediastinal mass was considered bulky if its maximum width on a posteroanterior chest $\mathrm{x}$-ray was equal to or greater than one third of the internal transverse diameter of the thorax at the level of T5/T6; any tumor masses larger than $5 \mathrm{~cm}$ at any extramediastinal site were also considered bulky. Patients were tested for serum creatinine, liver function tests (including hepatitis B virus antigens, and hepatitis $\mathrm{C}$ virus antibodies), uric acid, lactate dehydrogenase, and HIV, and patients had their cardiac function evaluated by echocardiography.

Responses were primarily evaluated by centrally reviewed PET scan after two cycles of doxorubicin, bleomycin, vinblastine, and dacarbazine (ABVD); further CT and PET evaluation was performed at the end of the sixth cycle of ABVD or, alternatively, after the fourth ifosfamide, gemcitabine, and vinorelbine (IGEV) course and at the end of any transplantation procedure. The depth of response was graded according to the revised response criteria for malignant lymphomas. ${ }^{23}$ A complete response was defined as the complete disappearance of all detectable clinical evidence of disease and disease-related symptoms, if present before therapy, with residual masses of any size being permitted if the scan was PET negative. Partial response was defined in case of at least a 50\% decrease in the sum of the products of the diameters (SPD) of up to six dominant masses without any increase in the size of other nodes, liver, or spleen. Moreover, it was required that splenic and hepatic nodules regressed by at least 50\% in their SPD, with the PET scan still positive in at least one prior involved site. The disease was considered stable if the patient failed to attain the criteria needed for a complete response or partial response, without fulfilling those for progressive disease. Progressive disease was defined as the appearance of any new lesion of more than $1.5 \mathrm{~cm}$ in any axis, even if other lesions were decreasing in size, or as an increase of at least $50 \%$ in the SPD of any previously involved node (or splenic or hepatic nodes). Any increased PET uptake in a previously unaffected site required confirmation with other modalities. Follow-up consisted of clinical and laboratory evaluation every 4 months after the end of the treatment program; CT and PET scans were performed yearly for the first 5 years of follow-up.

PET protocol. FDG PET scans were performed with modern full-ring dedicated PET/CT scanners. For each patient, baseline and follow-up scans were performed at the same nuclear medicine center and with the same instrument. The quality of the studies was guaranteed by quality control processes at each center by means of daily quality control measures and/or daily setup and/or tuning and periodical tests of PET performances according to manufacturers' recommendations and internal procedures. Inspection of uniformity and quantitative accuracy of the reconstructed image were considered to identify technical failures that were not detected by using the routine daily quality control procedures. In addition, sinogram data were visually inspected to check for detector failures. Performance Measurements of Positron Emission Tomographs (National Electrical Manufacturers Association NU2-2001) were scheduled at each PET site according to local procedures. Maintenance of all the other devices involved (eg, dose calibrators, well counters, clocks) was performed according to manufacturers' recommendations.

A dose of approximately 185 to $550 \mathrm{MBq}$ was administered intravenously as a bolus according to routine clinical acquisition protocol for the specified PET scanner. A whole-body acquisition with attenuation correction and with emission scan was performed 60 to 90 minutes after injection, starting from the groin up to the ears. The recommended interval between FDG administration and the start of acquisition is 60 minutes in the latest version of European Association of Nuclear Medicine Procedures Guidelines (Boellaard, et al: Eur J Nucl Med Mol Imaging 37:181-200, 2010). However, this recommended interval may change in clinical trials, depending on disease and study aims: times to scan of 60 to 90 minutes, as stated in the study protocol, have in fact been considered a reasonable interval, given the daily work-up of each center and given that uptake curves seem to become 
flatter at 60 to 90 minutes after injection (Shankar, et al: J Nucl Med 47:1059-1066, 2006; Lowe, et al: J Nucl Med 36:883-887, 1995; Hutchings, et al: Blood 107:52-59, 2006; Castellucci, et al: Eur J Nucl Med Mol Imaging 32:749-756, 2005). The time for bed position depended on the PET machine used and was left open. Scans corrected for decay, body weight, and administered activity were reconstructed by using iterative algorithms.

PET results have been scored according to a two-point visual scoring system (0: negative, normal, minimal residual uptake just below background, benign; 1: positive, malignant). Mediastinal uptake was used as a reference. In addition, all PET2-positive scans were reviewed by a board of three independent reviewers who were specifically recruited for this purpose to attribute the Deauville score. $^{25}$

Statistical analysis. Progression-free survival (PFS) comparison between positive and negative positron emission tomography evaluation after two cycles of chemotherapy (PET2) patients was performed by using a Cox proportional hazard model adjusting for age, sex, histology, increased lactate dehydrogenase, stage, systemic symptoms, and bulky disease.

\section{Results}

Survival analysis. Regarding patients with stage III to IV disease, the 2-year PFS was 79\% (95\% CI, 74\% to 82\%) for the entire population and $76 \%(95 \%$ CI, $65 \%$ to $85 \%)$ for the PET2-positive patients. In patients with stage II disease, the 2-year PFS was $83 \%$ ( $95 \%$ CI, $73 \%$ to $90 \%$ ) for the entire population and $76 \%$ (95\% CI, $48 \%$ to $90 \%$ ) for the PET2-positive patients. 